\title{
The HOMA-IR Performance to Identify New Diabetes Cases by Degree of Urbanization and Altitude in Peru: The CRONICAS Cohort Study
}

\author{
Rodrigo M. Carrillo-Larco $\mathbb{D}^{1,2}$ J. Jaime Miranda, ${ }^{1,3}$ Robert H. Gilman, ${ }^{1,4,5}$ \\ William Checkley, ${ }^{1,6}$ Liam Smeeth, ${ }^{1,7}$ Antonio Bernabe-Ortiz ${ }^{10},{ }^{1,7}$ \\ and CRONICAS Cohort Study Group ${ }^{1}$
}

\author{
${ }^{1}$ CRONICAS Center of Excellence in Chronic Diseases, Universidad Peruana Cayetano Heredia, Lima, Peru \\ ${ }^{2}$ Department of Epidemiology and Biostatistics, School of Public Health, Imperial College London, London, UK \\ ${ }^{3}$ Department of Medicine, School of Medicine, Universidad Peruana Cayetano Heredia, Lima, Peru \\ ${ }^{4}$ Department of International Health, Bloomberg School of Public Health, Johns Hopkins University, Baltimore, USA \\ ${ }^{5}$ Área de Investigación y Desarrollo, AB PRISMA, Lima, Peru \\ ${ }^{6}$ Division of Pulmonary and Critical Care, School of Medicine, Johns Hopkins University, Baltimore, USA \\ ${ }^{7}$ Faculty of Epidemiology and Population Health, London School of Hygiene and Tropical Medicine, London, UK
}

Correspondence should be addressed to Rodrigo M. Carrillo-Larco; rodrigo.carrillo@upch.pe

Received 28 April 2018; Revised 20 September 2018; Accepted 4 October 2018; Published 16 December 2018

Academic Editor: Antonio Brunetti

Copyright (c) 2018 Rodrigo M. Carrillo-Larco et al. This is an open access article distributed under the Creative Commons Attribution License, which permits unrestricted use, distribution, and reproduction in any medium, provided the original work is properly cited.

\begin{abstract}
Aims. Prognostic thresholds to identify new type 2 diabetes mellitus (T2DM) cases using the HOMA-IR have not been defined. We studied the HOMA-IR performance to identify incident T2DM cases and to assess if the thresholds varied according to urbanization and altitude in Peru. Methods. Longitudinal analysis. The outcome was incident T2DM cases: self-report diagnosis and fasting glucose. The exposure was the HOMA-IR. Receiver operating characteristic (ROC) curves were plotted, and the area under the ROC curve (AUC) was estimated with 95\% confidence intervals (95\% CIs). Results are presented overall and stratified by study site (Lima, Tumbes, urban Puno, and rural Puno), rurality (urban, semiurban, and rural), and altitude (low and high). Results. A total of 3120 participants (mean age: 55.6 years, $51.2 \%$ females) contributed data to this analysis. The median baseline HOMA-IR was 1.7 (IQR 1.0-2.9), with median values ranging from 1.1 in rural Puno to 2.0 in Lima and Tumbes $(p<0.001)$. Overall for incident T2DM, the AUC was 0.69 (95\% CI: $0.64-0.74)$ with an empirical threshold of 2.8 yielding a positive likelihood ratio of 2.30 and a negative one of 0.61 ; the positive and negative predictive values were $14.6 \%$ and $95.7 \%$, respectively. The empirical thresholds varied within the variables of interest, for example, from 0.9 in urban Puno to 2.9 in Lima. Conclusions. Using the HOMA-IR to identify incident T2DM cases seems to yield moderate accuracy. The HOMA-IR could help improve identifying people at high risk of T2DM.
\end{abstract}

\section{Introduction}

Type 2 diabetes mellitus (T2DM) has become one of the largest health problems in the world. It is among the top causes of disability-adjusted life years as well as years lived with disability, particularly in middle- and high-income countries $[1,2]$. The scenario is not different in Peru, where population-based studies in several areas reported a 7\% prevalence $[3,4]$ and an incidence of two new cases per one hundred person-years [4, 5]. These international and national figures pinpoint the value of T2DM prevention and early diagnosis.

An important approach to prevent or delay the T2DM burden is to identify those individuals at high risk of T2DM. Therefore, tools to identify T2DM cases, taking advantage of available resources and which are reliable in a target population, are needed. Laboratory facilities, along with their processes and logistics, are known by health 
systems in low- and middle-income countries, even though they may not be available in all health facilities. Identifying laboratory-based tests for early identification of T2DM may be an important asset and can potentiate the use of available resources instead of introducing completely new approaches. In this line, the homeostasis model assessment for insulin resistance (HOMA-IR), which combines fasting glucose and insulin, could be an option [6-10]. The HOMA-IR assesses the function of $\beta$-cells and thus informs physiological changes that occur before the onset of T2DM (i.e., precursor of T2DM) [11, 12], which makes this test a potential tool to identify people at high risk of developing T2DM in the near future. In addition, HOMA-IR thresholds to identify T2DM cases have not been established, and neither have they been studied in populations living in places at different stages of urbanization or altitude above the sea level. Consequently, this study is aimed at determining the HOMA-IR performance to identify incident T2DM cases in four areas in Peru. This study is aimed at providing evidence to further use existing laboratory tests-insulin and glucose-that could be available in health facilities.

\section{Methods}

2.1. Study Design. This is a longitudinal analysis using data of the CRONICAS Cohort Study [13]. Briefly, the CRONICAS Cohort Study is a population-based prospective cohort which started in September 2010. To date, two follow-up rounds have been conducted, fifteen and thirty months, on average, after the baseline recruitment.

2.2. Study Scenario. The CRONICAS Cohort Study was conducted in four Peruvian areas: Lima, Tumbes, rural Puno, and urban Puno [13]. These sites have different socioeconomic and geographical features. Lima is a highly urbanized metropolis at sea level. Tumbes is a semiurban area at sea level. Puno is a city at 3825 meters above the sea level, from which two different sites - urban and rural-were studied.

National statistics rank Lima with the highest human development index (0.63), followed by Tumbes (0.52) and then Puno (0.39) [14]. The $62.6 \%$ of the population in Lima had any health insurance, and this figure for Tumbes and Puno was $68.7 \%$ and $60.7 \%$, respectively [15]. The illiteracy frequency for people aged $\geq 15$ years in these settings was $2.3 \%$ in Lima, $3.0 \%$ in Tumbes, and $9.4 \%$ in Puno [16], whereas life expectancy was 77 years in Lima, 74 years in Tumbes, and 70 years in Puno [17].

2.3. Study Population. At baseline, the CRONICAS Cohort Study aimed for a sex- and age- (35-44, 45-54, 55-64, and $\geq 65$ years) stratified random sample of at least 1000 people in each study setting (500 in urban and rural Puno) [13]. An updated census was used to identify eligible subjects at each site. Accounting for all study locations, 11,544 people were identified, 4325 were enrolled, and 3741 were surveyed. At baseline, 3601 people were available with complete questionnaires. Regarding the follow-up rounds, 2892 (80.3\%) individuals were recontacted in the first follow-up, while in the second follow-up, this number was 2726 (75.7\%) people.
Because this work focused on incident diabetes, prevalent cases were excluded.

\section{Variables}

3.1. Outcome Variables. The outcome of interest was incident T2DM. T2DM new cases were identified at the second follow-up because of data availability. Having excluded T2DM cases at baseline (self-reported T2DM diagnosis made by a physician, currently taking medication for diabetes, and a fasting plasma glucose $\geq 126 \mathrm{mg} / \mathrm{dl}$ ), incident cases were identified if they were receiving medication for T2DM or had a fasting plasma glucose $\geq 126 \mathrm{mg} / \mathrm{dl}$. Blood samples were collected by a trained technician. Venous blood was withdrawn after a fasting period of eight to twelve hours. Plasma glucose was measured with an enzymatic colorimetric technique (GOD-PAP; modular P-E/Roche-cobas, Grenzach-Wyhlen, Germany) [13].

3.2. Exposure Variable. The exposure of interest was the HOMA-IR measured at baseline. This variable was computed as glucose $(\mathrm{mmol} / \mathrm{l})$ times insulin $(\mu \mathrm{U} / \mathrm{ml})$, all divided by 22.5 [(glucose $*$ insulin)/22.5] [18]. Blood samples for insulin were retrieved following the same procedures as explained above. Serum insulin was assessed using electrochemiluminescence (modular P-E/Roche-cobas) [13].

3.3. Other Variables. Other variables were assessed at baseline following standard questionnaires [13]: sex (men and women); age (35-44, 45-54, 55-64, and $\geq 65$ years); education ( $<7,7-11$, and $\geq 12$ years of education); study site (Lima, Tumbes, urban Puno, and rural Puno); altitude above the sea level (low altitude included Lima and Tumbes and high altitude encompassed Puno); rurality (urban for Lima as well as urban Puno, semiurban for Tumbes, and rural for rural Puno); and asset index, which is a numerical indicator based on household assets (in tertiles). Weight $(\mathrm{kg})$, height $(\mathrm{cm})$, and waist circumference $(\mathrm{cm})$ were measured with calibrated devices with the participants wearing light clothes and shoeless [13]. Hemoglobin A1C (HbA1c) was assessed with high-performance liquid chromatography (D10, Bio-Rad, Munich, Germany) [13]. For descriptive purposes, the HOMA-B was also estimated: $(20 \times$ insulin $) /($ glucose -3.5$)$ [18]. Follow-up time was estimated as the time difference between baseline and follow-up, except for people who developed T2DM whose follow-up time was deemed to be half of the observed follow-up time difference. This is a conservative approach taking into consideration that T2DM could have appeared anytime between assessments.

\section{Statistical Analysis}

The analyses were conducted with STATA v13.0 (StataCorp, College Station, TX, USA). For descriptive purposes, numerical variables were summarized with means along with standard deviations or medians with the corresponding interquartile range (IQR). Categorical variables were summarized using proportions. To compare numeric variables according to other categorical variables, the Wilcoxon ranksum and the Kruskal-Wallis rank tests were used. For 
comparison of categorical variables, the chi-squared test was conducted. To estimate the $p$ value for trend, the Cuzick test (nptrend command) was used. The receiver operating characteristic (ROC) curve was plotted, and the area under the ROC curve (AUC) was estimated using the roctab command. The AUC was compared among study sites, as well as by altitude and rurality using the roccomp command. Finally, empirical thresholds for the HOMA-IR to identify incident T2DM case were estimated with the cutpt command and the youden option; these calculations yielded the Youden's index, sensitivity, specificity, and the AUC for each given threshold. To estimate the $95 \%$ confidence interval (95\% CI) of each empirical threshold and its corresponding AUC, a postestimation bootstrap technique was followed assuming one thousand replications without replacement. The positive likelihood ratio was estimated as sensitivity over one minus specificity, whereas the negative likelihood ratio was defined as one minus sensitivity over specificity. The negative predictive value was estimated as [sensitivity $\times$ prevalence] divided by [sensitivity $\times$ prevalence $+(1-$ specificity $) \times(1-$ prevalence $)$ ]; the negative predictive value was computed as [specificity $\times(1-$ prevalence $)]$ divided by $[(1-$ sensitivity $) \times$ prevalence + specificity $\times(1-$ prevalence $)]$. In order to assess whether the expected (using the HOMA-IR as predictor) and observed number of diabetes cases matched across all doses of the exposure (the HOMA-IR divided in deciles), we used the Hosmer-Lemeshow chi-squared goodness of fit test; high $p$ values $(p>0.05)$ mean that the expected and observed numbers of cases are similar; thus, the predictor shows good calibration. To compare the AUC yielded for incident T2DM between the HOMA-IR and HbA1c, the roccomp command was used; this tests if the AUC is equal between assessment methods.

\section{Ethics}

The protocol of the CRONICAS Cohort Study was reviewed and approved by three independent Institutional Review Boards in Peru and in the USA: Universidad Peruana Cayetano Heredia and Associaion Benefica PRISMA in Lima, Peru, as well as by the Johns Hopkins University in Baltimore, USA. All participants gave verbal informed consent [13]. The research was conducted in accordance with the Helsinki declaration.

\section{Results}

6.1. Study Population at Baseline. There were 3601 subjects, though 481 (13.4\%) were excluded because of missing values in baseline glucose or insulin; there were no differences in gender and age, but excluded subjects were poorer and there were more excluded subjects in Puno (Supplementary Table 1).

At baseline, mean age was 55.6 (SD: 12.7) years, and $51.2 \%$ of the study sample was female. The prevalence of T2DM was $6.9 \%$ (95\% CI: 6.1\%-7.9\%). The median HOMA-IR was 1.7 (IQR 1.0-2.9) and the median HOMAB was 96.4 (IQR 59.5-149.7). Further details about these sociodemographic and clinical variables are shown in Table 1 , overall and by study site.

6.2. HOMA-IR: Characteristics at Baseline. Differences in the HOMA-IR by sociodemographic and clinical variables are shown in Table 2. Median values of the HOMA-IR varied among urban, semiurban, and rural sites: $1.8,2.0$, and 1.1 ( $p$ for trend $<0.001$ ), respectively. The higher the wealth index, the higher the HOMA-IR: median of 1.4 at the bottom tertile and of 2.0 at the top tertile ( $p$ for trend $<0.001$ ).

6.3. New Diabetes Cases. The mean follow-up time was 2.3 (SD: 0.5) years. Among those who did not have T2DM at baseline, 120 people developed T2DM. Subjects who developed T2DM at follow-up had higher HOMA-IR at baseline in comparison to those who did not develop T2DM $(p<0.001)$ : the median was 2.9 (IQR: $1.7-4.3$ ) and 1.7 (IQR: 1.0-2.7), respectively. A similar difference was retrieved when comparing these populations regarding fasting glucose at baseline $(p<0.001)$ : subjects who did not develop T2DM had a median glucose of $91.0 \mathrm{mg} / \mathrm{dl}$ (IQR: 85.0-97.9) and those who developed T2DM had a median glucose of $102.5 \mathrm{mg} / \mathrm{dl}$ (IQR: 91.7-116.0).

6.4. HOMA-IR: Diagnostic Performance for Incident Cases. The AUC for incident T2DM cases was 0.68 (95\% CI: 0.640.74 , Figure 1). The AUC for each study population is presented in Table 3, along with the corresponding empirical thresholds.

Empirical HOMA-IR thresholds were different among study sites, varying from 0.9 (95\% CI: $0.00-1.96)$ to 2.9 (95\% CI: 2.11-3.69) in urban Puno and Lima, respectively. Dissimilar thresholds were also found according to altitude: 2.8 (95\% CI: $2.20-3.48)$ at low and 1.2 (95\% CI: $0.62-1.71$ ) at high altitude, as well as according to rurality: 2.5 (95\% CI: $1.67-3.26)$ in urban, 2.8 (95\% CI: $1.80-3.88$ ) in semiurban, and 1.2 (95\% CI: 0.76-1.58) in rural areas. There was evidence of moderate calibration $(p=0.065)$, with good agreement (Figure 2) in the tenth decile (i.e., highest HOMA-IR level).

6.5. HOMA-IR in Comparison to HbA1c. The AUC was larger for HbA1c to predict new T2DM cases: 0.75 (95\% CI: 0.70$0.80, p=0.023)$ for HbAlc.

\section{Discussion}

7.1. Main Results. In a population-based study conducted in four Peruvian areas at different stages of urbanization as well as with different sociodemographic and health profiles, the HOMA-IR had moderate prediction accuracy for incident T2DM cases in terms of discrimination and calibration. The HOMA-IR thresholds for incident T2DM cases varied according to study site, altitude, and degree of urbanization. The HOMA-IR AUC was smaller in comparison to HbAlc, suggesting that the latter could be preferred to identify people at high risk of T2DM.

7.2. Comparison with Other Studies. Our AUC for incident T2DM was moderate, which could signal that these people 
TABLE 1: Sociodemographic and clinical characteristics of study participants at baseline and overall and according to study site.

\begin{tabular}{|c|c|c|c|c|c|c|}
\hline Variable & Overall & Lima & Urban Puno & Rural Puno & Tumbes & $p$ value \\
\hline Sex & $N=3118$ & $N=1031$ & $N=517$ & $N=539$ & $N=1031$ & 0.882 \\
\hline Female & 51.2 & 51.8 & 50.9 & 52.1 & 50.3 & \\
\hline Male & 48.8 & 48.2 & 49.1 & 47.9 & 49.7 & \\
\hline Age (years) & $N=3118$ & $N=1031$ & $N=517$ & $N=539$ & $N=1031$ & 0.934 \\
\hline $35-44$ & 24.3 & 24.0 & 24.8 & 22.8 & 25.3 & \\
\hline $45-54$ & 25.5 & 27.1 & 25.0 & 25.4 & 24.4 & \\
\hline $55-64$ & 25.4 & 25.2 & 25.7 & 25.4 & 25.3 & \\
\hline $65+$ & 24.8 & 23.8 & 24.6 & 26.4 & 25.0 & \\
\hline Wealth index & $N=3120$ & $N=1031$ & $N=517$ & $N=541$ & $N=1031$ & $<0.001$ \\
\hline Bottom & 31.6 & 12.0 & 23.2 & 71.5 & 34.5 & \\
\hline Middle & 33.9 & 37.0 & 26.1 & 26.1 & 39.0 & \\
\hline Top & 34.4 & 51.0 & 50.7 & 2.4 & 26.5 & \\
\hline Education & $N=3118$ & $N=1030$ & $N=517$ & $N=541$ & $N=1030$ & $<0.001$ \\
\hline$<7$ years & 45.9 & 42.8 & 14.7 & 62.9 & 55.6 & \\
\hline $7-11$ years & 32.9 & 39.7 & 27.3 & 30.5 & 30.3 & \\
\hline$\geq 12$ years & 21.2 & 17.5 & 58.0 & 6.7 & 14.1 & \\
\hline Waist circumference & $N=3115$ & $N=1031$ & $N=514$ & $N=540$ & $N=1030$ & $<0.001$ \\
\hline Mean (SD) & $91.9(11.0)$ & $92.4(10.5)$ & $92.6(10.8)$ & $85.0(11.0)$ & $94.6(10.2)$ & \\
\hline Body mass index & $N=3116$ & $N=1031$ & $N=514$ & $N=540$ & $N=1031$ & $<0.001$ \\
\hline Mean (SD) & $27.7(4.6)$ & $28.4(4.6)$ & $27.8(4.3)$ & $25.1(3.7)$ & $28.3(4.8)$ & \\
\hline HbAlc & $N=3119$ & $N=1031$ & $N=516$ & $N=541$ & $N=1031$ & \\
\hline Median (IQR) & $5.8(5.5-6.1)$ & $5.7(5.4-5.9)$ & $5.9(5.6-6.1)$ & $5.8(5.6-6.1)$ & $5.9(5.6-6.2)$ & $<0.001^{*}$ \\
\hline Diabetes & $N=3120$ & $N=1031$ & $N=517$ & $N=541$ & $N=1031$ & $<0.001$ \\
\hline Yes & 6.9 & 5.5 & 7.0 & 3.1 & 10.3 & \\
\hline HOMA-B & $N=3118$ & $N=1030$ & $N=517$ & $N=540$ & $N=1031$ & $<0.001^{*}$ \\
\hline Median (IQR) & $96.4(59.5-149.7)$ & $108.0(68.8-162.8)$ & $95.5(60.9-153.2)$ & $74.1(46.1-119.2)$ & $98.6(57.9-149.2)$ & \\
\hline HOMA-IR & $N=3120$ & $N=1031$ & $N=517$ & $N=541$ & $N=1031$ & \\
\hline Median (IQR) & $1.7(1.04-2.94)$ & $2.0(1.17-3.19)$ & $1.6(1.04-2.75)$ & $1.1(0.62-1.76)$ & $2.0(1.23-3.42)$ & $<0.001^{*}$ \\
\hline
\end{tabular}

Results are presented as percentages for categorical variables. $p$ values for categorical variables refer to the chi-squared test among study sites, whereas for numerical variables (waist circumference and body mass index) to the analysis of variance. ${ }^{*} p$ value refers to the Kruskal-Wallis equality-of-populations rank test among study sites.

are still on their way to T2DM, i.e., would develop T2DM in more than 2.3 years of follow-up. Another study with a median follow-up of nine years and using oral glucose tolerance test (OGTT) as the gold standard reported an AUC of $72 \%$ for women and $66 \%$ for men [7]. Although prospective evidence on the prediction accuracy of the HOMA-IR is still scarce and deserves further comprehensive scrutiny across populations and health backgrounds, the HOMA-IR appears to have a moderate performance to identify incident T2DM cases even after several years.

7.3. Result Interpretation. Including all the study population, the threshold for incident T2DM cases was 2.8. Other authors have suggested cutoffs of around 2.2 [7] and 2.0 [10]. These differences could be because of other determinants of insulin resistance, e.g., visceral fat, or because those populations have different T2DM profiles or are at an advanced stage of the nutritional transition. This would mean that populations with higher levels of these risk factors would experience detrimental effects at lower thresholds of insulin resistance [7]. Nevertheless, the distribution of body mass index and waist circumference was comparable among our study population and previous reports, suggesting that other variables would explain these differences, perhaps genetics or other unmeasured anthropometric features, such as skinfolds [7, 10, 19].

According to study site, the $95 \%$ CIs of some empirical thresholds overlap, especially the ones from sites in Puno and those of Lima and Tumbes. However, the results do not support using one threshold across all sites. Furthermore, the positive and negative likelihood ratios are also more similar between Lima and Tumbes and between Puno sites. These pairwise similarities could be explained by shared health profiles $[4,20]$. This pattern across study sites is also reflected in the empirical thresholds and their 95\% CIs according to rurality level: urban and semiurban sites showed 
TABLE 2: The baseline HOMA-IR according to sociodemographic variables.

\begin{tabular}{|c|c|c|}
\hline Variable & Median (IQR) & $p$ value \\
\hline Sex & & $<0.001$ \\
\hline Female & $2.0(1.2-3.4)$ & \\
\hline Male & $1.5(0.9-2.5)$ & \\
\hline Age (years) & & $<0.001 /<0.001$ \\
\hline $35-44$ & $1.8(1.1-2.9)$ & \\
\hline $45-54$ & $1.8(1.1-3.1)$ & \\
\hline $55-64$ & $1.9(1.2-3.3)$ & \\
\hline $65+$ & $1.5(0.8-2.6)$ & \\
\hline Asset index & & $<0.001 /<0.001$ \\
\hline Bottom & $1.4(0.8-2.4)$ & \\
\hline Middle & $1.8(1.1-3.0)$ & \\
\hline Top & $2.0(1.3-3.4)$ & \\
\hline Education & & $<0.001 /<0.001$ \\
\hline$<7$ years & $1.7(1.0-2.9)$ & \\
\hline $7-11$ years & $1.7(1.0-2.9)$ & \\
\hline$\geq 12$ years & $2.0(1.2-3.1)$ & \\
\hline Study site & & $<0.001$ \\
\hline Lima & $2.0(1.2-3.2)$ & \\
\hline Urban Puno & $1.6(1.0-2.8)$ & \\
\hline Rural Puno & $1.1(0.6-1.8)$ & \\
\hline Tumbes & $2.0(1.2-3.4)$ & \\
\hline Altitude & & $<0.001 /<0.001$ \\
\hline Low & $2.0(1.2-3.4)$ & \\
\hline High & $1.3(0.8-2.2)$ & \\
\hline Rurality & & $<0.001 /<0.001$ \\
\hline Urban & $1.8(1.1-3.0)$ & \\
\hline Semiurban & $2.0(1.2-3.4)$ & \\
\hline Rural & $1.1(0.6-1.8)$ & \\
\hline Diabetes & & $<0.001$ \\
\hline No & $1.6(1.0-2.7)$ & \\
\hline Yes & $4.8(2.6-8.5)$ & \\
\hline
\end{tabular}

Results are presented as median (IQR). $p$ values refer to the Wilcoxon ranksum or to the Kruskal-Wallis tests for independence; if there is a second $p$ value, it corresponds to the $p$ value for trend.

95\% CIs that closely overlap, whereas rural sites have different ranges. The evidence when looking at altitude above the sea level appears to be more conclusive and suggests that Puno (i.e., high-altitude sites) needs a different threshold than Lima and Tumbes.

Across sites and empirical thresholds, the negative predictive values were above $95 \%$, suggesting that this test could be used to "rule out" future cases or at least to signal people that could have a less thorough follow-up with other diabetes biomarkers in the near future. Even though all positive likelihood ratio estimates were small and would not suppose a large change in the pretest probability, the largest estimate was found in Tumbes. This could highlight the unhealthy profile of this population $[4,20]$, thus the already high probability of finding diabetes cases among them. On the other hand, and consistent with the high negative predictive values,

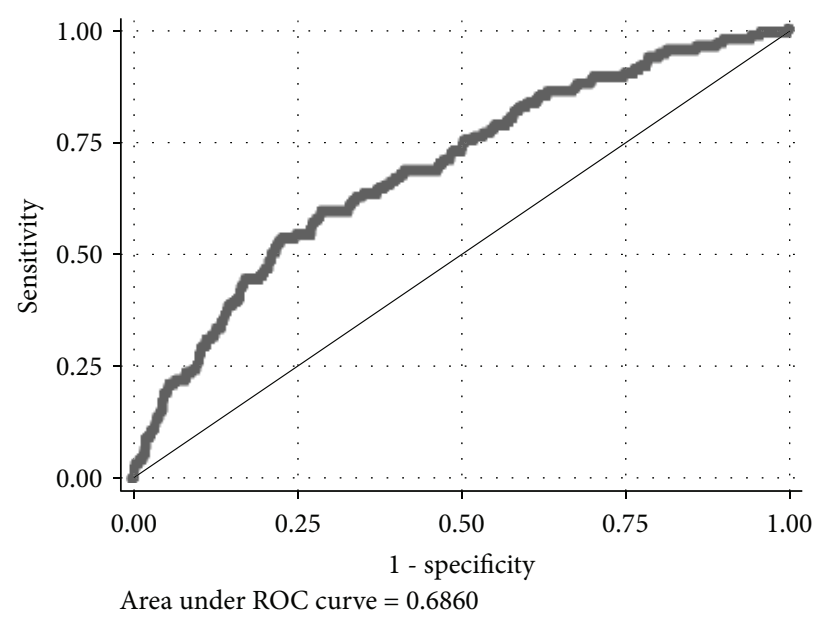

FIgURe 1: ROC for the HOMA-IR and incident diabetes cases at follow-up.

there were relevant negative likelihood ratio estimates in Puno. In other words, and consistent with an overall healthier profile of this population, someone with a negative test result would have low probabilities of becoming diabetic in the near future.

7.4. Public Health Relevance. Current diabetes guidelines recommend screening for all people above 45 years of age or if they have risk factors for diabetes and to repeat the screening within the next three years, though this could be shorter depending on initial results [21]. Our findings could support testing a person with a negative HOMA-IR result in more than a year, but less than 2.3 years (follow-up period). Our results indicated large negative likelihood ratio and negative predictive value estimates across sites. These metrics suggest that it is unlikely for a person with a negative result to develop T2DM in the next 2.3 years, albeit this evidence should be interpreted in light with the study limitations and according to each patient's characteristics as well as the epidemiological profile of the population. Future studies should verify our results and test their implementation in clinical practice.

Current massive testing with the HOMA-IR could be limited by economic shortcuts, which in turn reflects a slightly less easy laboratory process. In a Peruvian public hospital, a fasting glucose, an OGTT, and an insulin test cost 1.0 US\$, 2.8 US\$, and 3.4 US\$, respectively [22]. In a thirtymonth period, a given subject could have three fasting glucose tests, totaling 3.0 US\$. If, however, this subject were tested with the HOMA-IR (4.4 US\$), he could have undergone one test only in the same period. This may not be cost-effective (3.0 US\$ vs. 4.4 US\$), also when compared to $\mathrm{HbA1c}$, which yielded better AUC in our results. Nevertheless, the HOMA-IR could be useful in rural areas where laboratories are not available and thus people have additional expenses (e.g., time and transportation) to get screening; thereby, people from rural areas could commute (although this consumes additional time and costs), have a HOMA-IR test, and should it be a negative result, then follow-up 


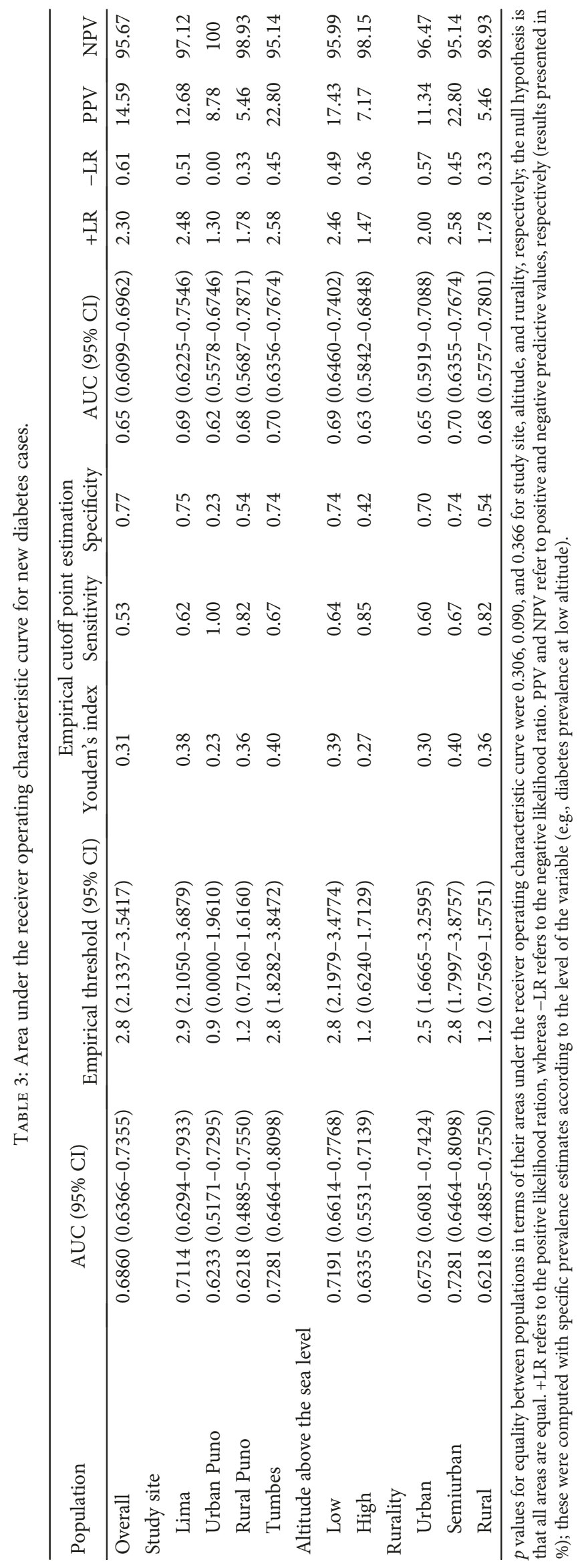




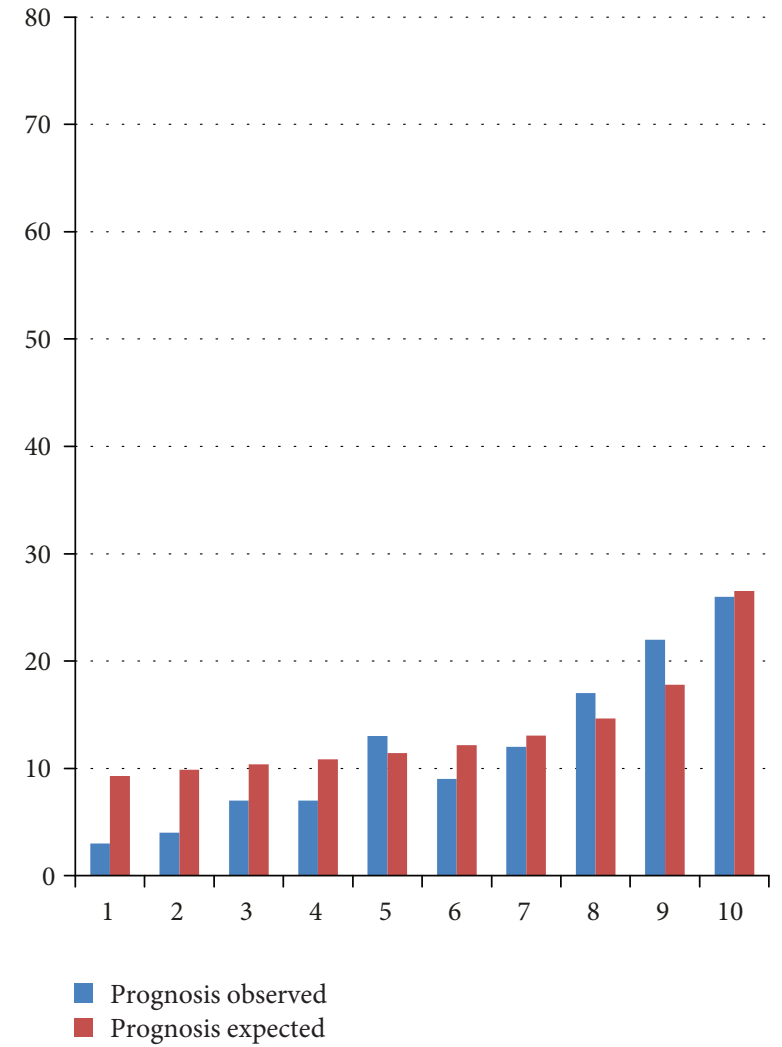

FIGURE 2: Agreement between expected and observed incident diabetes cases.

evaluations could be delayed one or two years, yet no further than 2.3 years. Our result yielded strong negative predictive values, suggesting that a person with a negative result has low chances of developing T2DM; hence, follow-up visits could be less often than on a yearly basis, as long as the overall health profile allows. These are speculative arguments, and the HOMA-IR requires further studies before its enactment is strongly suggested or discouraged.

Other blood-based test is $\mathrm{HbAlc}$, which, in comparison to the HOMA-IR, appears to be more accurate, cheaper, and less cumbersome because it does not require a fasting period. Notwithstanding, $\mathrm{HbAlc}$ needs high-quality processing to secure consistent results and could also have some limitations at high altitude [23]. Other studies have assessed HbAlc to predict T2DM cases. In China, for example, the AUC was $64.5 \%$ (medial follow-up: 4.6) [24], while in rural Korea, it was $74 \%$ (median follow-up: 4.0 years) [25]. Our AUC for HbA1c (and AUC) for incident T2DM cases was in between these figures.

On the other hand, the overall results at the proposed threshold signal a higher specificity than sensitivity. This means that it would be more likely to identify healthy people (i.e., without risk of developing T2DM). This has public health relevance because knowing who is not likely to develop T2DM would allow allocating (limited) resources to high-risk individuals. Furthermore, the ratio between true positive and false negative, as derived from the overall sensitivity and specificity estimates, is 1.13 . This reveals that for each 10 false negatives, roughly 11 true positive cases would be identified.

7.5. Strengths and Limitations. This study benefits from a relatively large sample size, but most importantly from including participants from study sites with different sociodemographic and health characteristics. We analyzed prospective data, which allowed us to study the short-term effect of the HOMA-IR to identify new T2DM cases. Nevertheless, limitations of the study need to be acknowledged too. First, our T2DM diagnosis was based on fasting glucose. If in addition to or in replace of this method we have used another one, namely, OGTT, we could have detected more T2DM cases. However, if this were the case, our results are still conservative and there could have been a larger AUC. Second, most of the subjects excluded from the analysis were from Puno. Results for this site should thus be interpreted cautiously. Third, some subjects were lost to follow-up; thus, there could have been more new cases. Our results warrant further verification, i.e., external validation. Fourth, although differences across sites could be attributed to different laboratory methods, all samples were analyzed by the same laboratory minimizing this potential bias.

\section{Conclusions}

Using the HOMA-IR to identify incident T2DM cases seems to yield moderate accuracy. Given the increasing burden of T2DM, actions need to be taken to improve the early identification of these patients. Using available laboratory facilities to assess the HOMA-IR could have positive results.

\section{Data Availability}

The data used to support the findings of this study are available from the corresponding author upon request.

\section{Conflicts of Interest}

The authors declare that they have no conflicts of interest.

\section{Authors' Contributions}

RMC-L conceived the idea and conducted the analysis with support of AB-O. RMC-L drafted the first version of the manuscript. All other authors gave critical insights and edits to improve the work. All authors accepted the final version. Juan P. Casas, George Davey Smith, Shah Ebrahim, Luis Huicho, Germán Málaga, Víctor M. Montori, Gregory B. Diette, Fabiola León-Velarde, María Rivera, Robert A. Wise, and Katherine Sacksteder are members of the CRONICAS Cohort Study Group.

\section{Acknowledgments}

Special thanks are due to all field teams for their commitment and hard work, especially to Lilia Cabrera, Rosa Salirrosas, Viterbo Aybar, Sergio Mimbela, and David Danz, for their leadership in each of the study sites, as well as Marco Varela for the data coordination. The CRONICAS Cohort Study was 
funded with federal funds from the NHLBI, National Institutes of Health, U.S. Department of Health and Human Services, under Contract number HHSN268200900033C. Liam Smeeth is a senior clinical fellow and Antonio Bernabe-Ortiz is a research training fellow in Public Health and Tropical Medicine (103994/Z/14/Z), both funded by the Wellcome Trust.

\section{Supplementary Materials}

Table S1: comparison between included and excluded subjects in the analysis. (Supplementary Materials)

\section{References}

[1] "Global, regional, and national disability-adjusted life-years (DALYs) for 333 diseases and injuries and healthy life expectancy (HALE) for 195 countries and territories, 1990-2016: a systematic analysis for the Global Burden of Disease Study 2016," Lancet, vol. 390, no. 10100, pp. 1260-1344, 2017.

[2] "Global, regional, and national incidence, prevalence, and years lived with disability for 328 diseases and injuries for 195 countries, 1990-2016: a systematic analysis for the Global Burden of Disease Study 2016," Lancet, vol. 390, no. 10100, pp. 1211-1259, 2017.

[3] S. N. Seclen, M. E. Rosas, A. J. Arias, E. Huayta, and C. A. Medina, "Prevalence of diabetes and impaired fasting glucose in Peru: report from PERUDIAB, a national urban population-based longitudinal study," BMJ Open Diabetes Research \& Care, vol. 3, no. 1, article e000110, 2015.

[4] A. Bernabé-Ortiz, R. M. Carrillo-Larco, R. H. Gilman et al., "Geographical variation in the progression of type 2 diabetes in Peru: the CRONICAS Cohort Study," Diabetes Research and Clinical Practice, vol. 121, pp. 135-145, 2016.

[5] S. N. Seclen, M. E. Rosas, A. J. Arias, and C. A. Medina, "Elevated incidence rates of diabetes in Peru: report from PERUDIAB, a national urban population-based longitudinal study," BMJ Open Diabetes Research \& Care, vol. 5, no. 1, article e000401, 2017.

[6] K. J. Yun, K. Han, M. K. Kim et al., "Insulin resistance distribution and cut-off value in Koreans from the 2008-2010 Korean National Health and Nutrition Examination Survey," PLoS One, vol. 11, no. 4, article e0154593, 2016.

[7] A. Ghasemi, M. Tohidi, A. Derakhshan, M. Hasheminia, F. Azizi, and F. Hadaegh, "Cut-off points of homeostasis model assessment of insulin resistance, beta-cell function, and fasting serum insulin to identify future type 2 diabetes: Tehran Lipid and Glucose Study," Acta Diabetologica, vol. 52, no. 5, pp. 905-915, 2015.

[8] H.-Q. Qu, Q. Li, A. R. Rentfro, S. P. Fisher-Hoch, and J. B. McCormick, "The definition of insulin resistance using HOMA-IR for Americans of Mexican descent using machine learning," PLoS One, vol. 6, no. 6, article e21041, 2011.

[9] Q. Tang, X. Li, P. Song, and L. Xu, "Optimal cut-off values for the homeostasis model assessment of insulin resistance (HOMA-IR) and pre-diabetes screening: developments in research and prospects for the future," Drug Discoveries \& Therapeutics, vol. 9, no. 6, pp. 380-385, 2015.

[10] C. H. Lee, A. Z. L. Shih, Y. C. Woo et al., "Optimal cut-offs of homeostasis model assessment of insulin resistance (HOMAIR) to identify dysglycemia and type 2 diabetes mellitus: a 15-year prospective study in Chinese," PLoS One, vol. 11, no. 9 , article e0163424, 2016.

[11] A. G. Tabak, M. Jokela, T. N. Akbaraly, E. J. Brunner, M. Kivimaki, and D. R. Witte, "Trajectories of glycaemia, insulin sensitivity, and insulin secretion before diagnosis of type 2 diabetes: an analysis from the Whitehall II study," Lancet, vol. 373, no. 9682, pp. 2215-2221, 2009.

[12] A. Hulman, R. K. Simmons, E. J. Brunner et al., "Trajectories of glycaemia, insulin sensitivity and insulin secretion in South Asian and white individuals before diagnosis of type 2 diabetes: a longitudinal analysis from the Whitehall II cohort study," Diabetologia, vol. 60, no. 7, pp. 1252-1260, 2017.

[13] J. J. Miranda, A. Bernabe-Ortiz, L. Smeeth, R. H. Gilman, W. Checkley, and CRONICAS Cohort Study Group, "Addressing geographical variation in the progression of non-communicable diseases in Peru: the CRONICAS cohort study protocol," BMJ Open, vol. 2, no. 1, article e000610, 2012.

[14] Programa de las Naciones Unidas para el Desarrollo (PNUD), "Informe sobre Desarrollo Humano Perú 2013. Cambio climático y territorio: desafíos y respuestas para un futuro sostenible," 2013, http://www.pe.undp.org/content/peru/es/home/ library/poverty/Informesobredesarrollohumano2013/ IDHPeru2013.html.

[15] Instituto Nacional de Estadistica (INEI), “Acceso a seguro de salud," 2013, https://www.inei.gob.pe/estadisticas/indicetematico/access-to-health-insurance/.

[16] Instituto Nacional de Estadistica (INEI), "Tasa de analfabetismo de la población de 15 y más años de edad, según grupos de edad y ámbitos geográficos," 2015, https://www.inei.gob.pe/ estadisticas/indice-tematico/sociales/.

[17] Instituto Nacional de Estadistica (INEI), "Indicadores demografico por departamento," 2015, https://www.inei.gob.pe/ estadisticas/indice-tematico/poblacion-y-vivienda/.

[18] T. M. Wallace, J. C. Levy, and D. R. Matthews, "Use and abuse of HOMA modeling," Diabetes Care, vol. 27, no. 6, pp. 14871495, 2004.

[19] C. R. Pullinger, I. D. Goldfine, S. Tanyolaç et al., "Evidence that an $H M G A 1$ gene variant associates with type 2 diabetes, body mass index, and high-density lipoprotein cholesterol in a Hispanic-American population," Metabolic Syndrome and Related Disorders, vol. 12, no. 1, pp. 25-30, 2014.

[20] A. Bernabé-Ortiz, R. M. Carrillo-Larco, R. H. Gilman et al., "Impact of urbanisation and altitude on the incidence of, and risk factors for, hypertension," Heart, vol. 103, no. 11, pp. 827-833, 2017.

[21] American Diabetes Association, "2. Classification and diagnosis of diabetes," Diabetes Care, vol. 40, Supplement 1, pp. S11-S24, 2017.

[22] Hospital Nacional Hipolito Unanue, “Tarifario 2016,” 2017, https://goo.gl/JH7iHp.

[23] J. C. Bazo-Alvarez, R. Quispe, T. D. Pillay et al., "Glycated haemoglobin $\mathrm{HbA}_{1 \mathrm{c}}$ and fasting plasma glucose relationships in sea-level and high-altitude settings," Diabetic Medicine, vol. 34, no. 6, pp. 804-812, 2017.

[24] P. Vijayakumar, R. G. Nelson, R. L. Hanson, W. C. Knowler, and $\mathrm{M}$. Sinha, " $\mathrm{HbA}_{1 \mathrm{c}}$ and the prediction of type 2 diabetes in children and adults," Diabetes Care, vol. 40, no. 1, pp. 1621, 2017.

[25] B. M. Song, H. C. Kim, J. Y. Lee et al., "Performance of $\mathrm{HbA}_{1 \mathrm{c}}$ for the prediction of diabetes in a rural community in Korea," Diabetic Medicine, vol. 32, no. 12, pp. 1602-1610, 2015. 


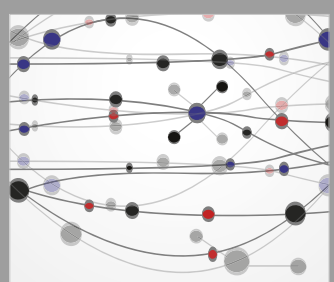

The Scientific World Journal
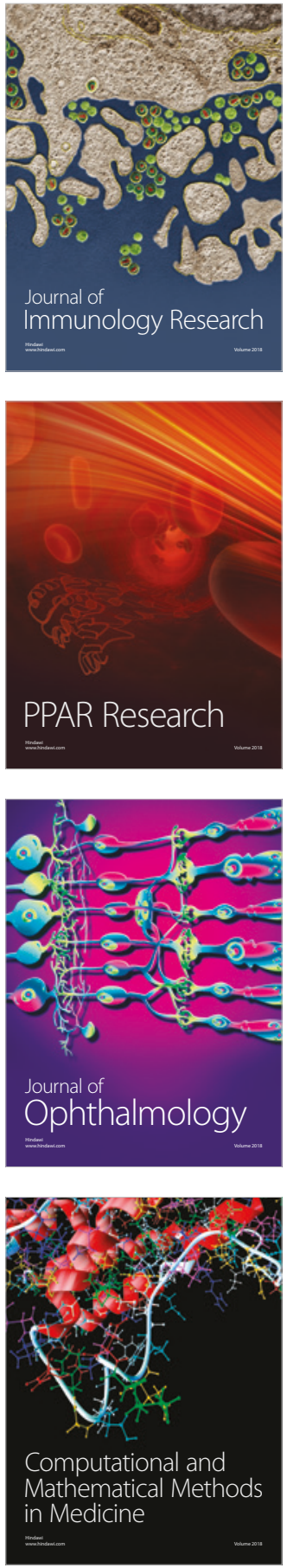

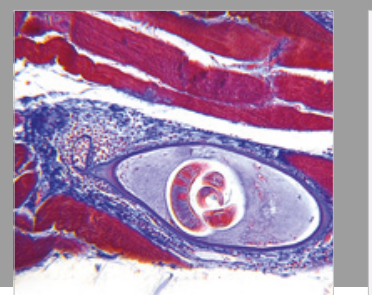

Gastroenterology Research and Practice

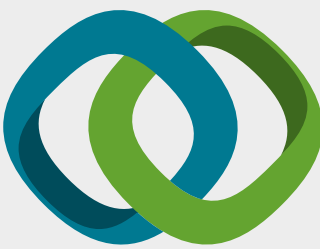

\section{Hindawi}

Submit your manuscripts at

www.hindawi.com
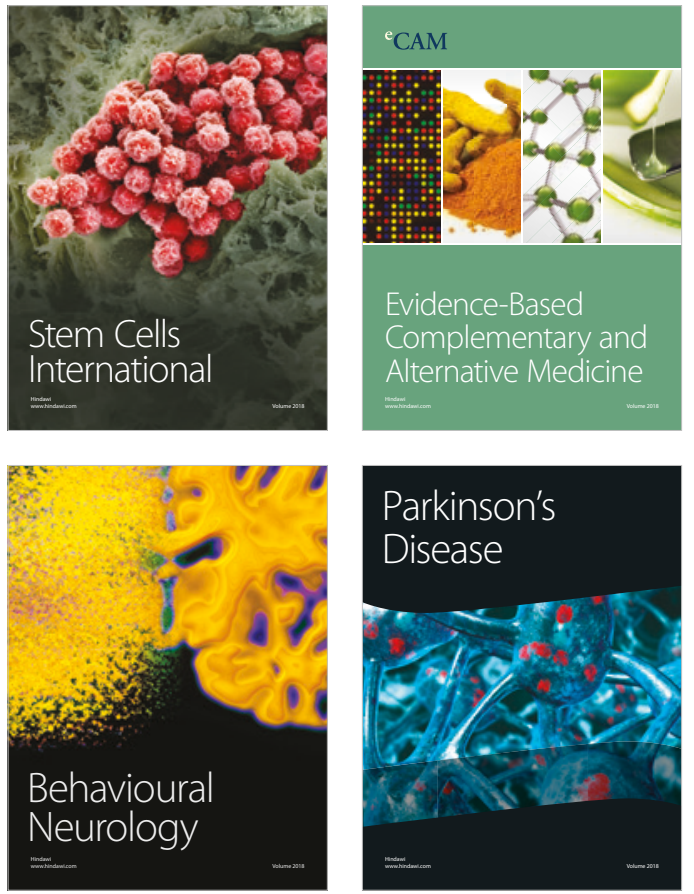

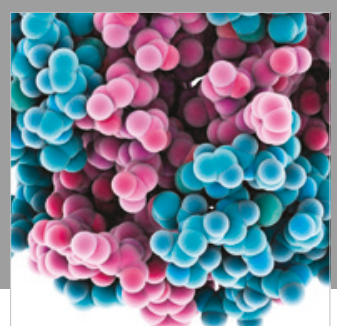

ournal of

Diabetes Research

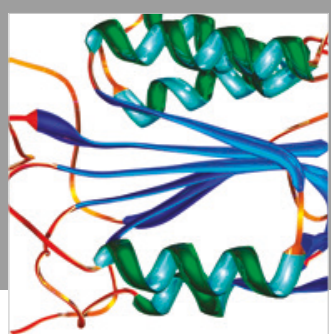

Disease Markers
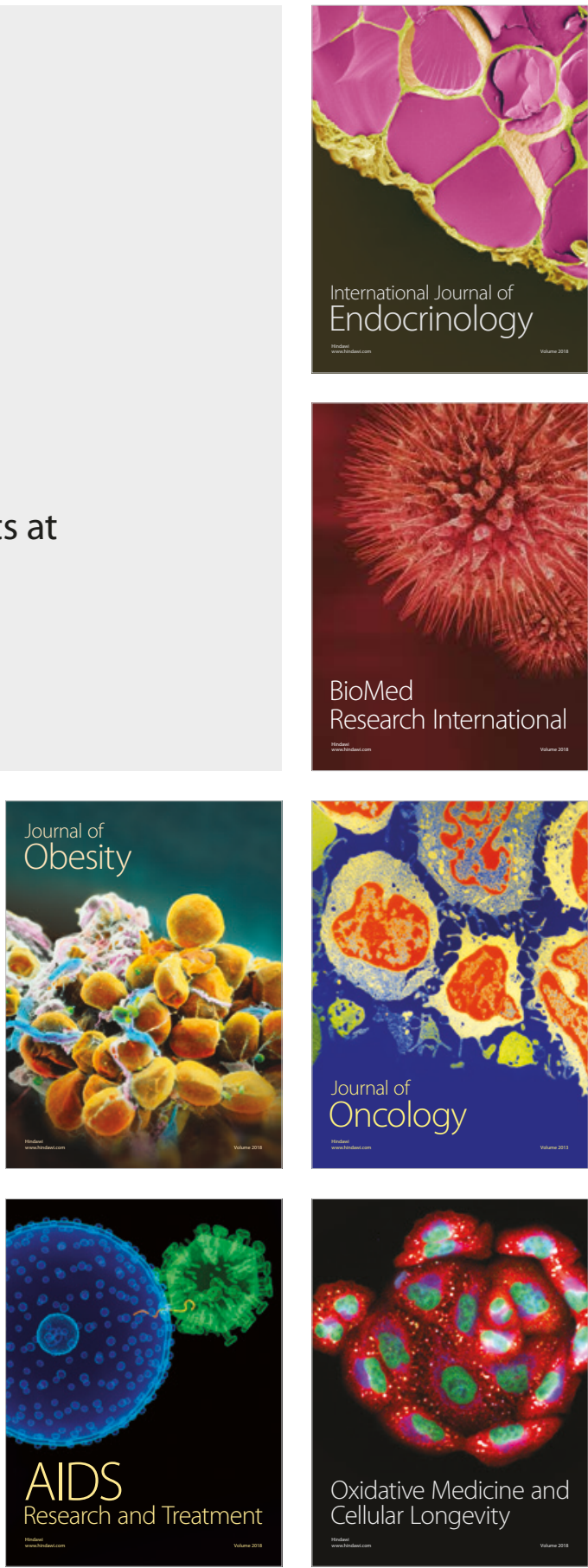